\title{
CORRELACIÓN CITOHISTOLÓGICA DE LESIONES ESCAMOSAS INTRAEPITELALES DE CUEШO UTERINO, EN LA UNIDAD DE PATOLOGÍA CERVICAL DEL HOSPITAL NAVAL DE TALCAHUANO
}

\author{
Luis Cuitiño G. ${ }^{1}$, Fernando Tirapegui S. ${ }^{a}$, Luis Torres Y.2, Rodrigo Klaassen P. ${ }^{2}$, Rina \\ Naveas G. ${ }^{b}$, Jessica Martínez C. $^{c}$ \\ ${ }^{1}$ Departamento de Ginecología y Obstetricia, Escuela de Medicina, Universidad San Sebastián. 2Unidad de Patología \\ Cervical, Servicio de Ginecología y Obstetricia, Hospital Naval Talcahuano.
}

anterno de Medicina, Universidad San Sebastián. ' ${ }^{\text {bestadístico. }}$ Matrona.

\section{RESUMEN}

Objetivo: Evaluar la calidad del laboratorio de citología cervical para LIE de bajo y alto grado del Hospital Naval de Talcahuano a través de la correlación citohistológica. Material y Método: Estudio retrospectivo, descriptivo y analítico. Se revisaron 422 historias clínicas de pacientes ingresadas a la Unidad de Patología Cervical (UPC) del Hospital Naval de Talcahuano, en el período 1994-2004. En 204 casos hubo citología positiva para LIE, 83,3\% tenían una biopsia bajo visión colposcópica, y 75\% tenían una biopsia por escisión con asa electroquirúrgica. Como clasificación diagnóstica se empleó el sistema Bethesda. Resultados: No hubo diferencia significativa entre la media de edad en las pacientes que presentaban LIE de bajo y alto grado, tanto para el grupo con biopsia bajo visión colposcópica como para el grupo con biopsia con asa electroquirúrgica. En el grupo con biopsia bajo visión colposcópica hubo coincidencia citohistológica en $67,1 \%$ para LIE bajo grado, y $67,1 \%$ para LIE alto grado. En el grupo con biopsia con asa electroquirúrgica hubo coincidencia citohistológica en $59,7 \%$ para LIE bajo grado, y $73,7 \%$ para LIE alto grado. Existe correlación entre citología para LIE e histopatología tanto de biopsia bajo visión colposcópica, como por escisión con asa electroquirúrgica $(\mathrm{p}<0,001)$. Discusión: El promedio de edad de los pacientes que portaban un LIE bajo grado en ambos grupos de estudio fue superior a las series nacionales e internacionales. En la UPC del Hospital Naval (T) el screening citológico es confiable para LIE de bajo y alto grado.

\section{PALABRAS CLAVES: Correlación citohistológica, LIE, pesquisa citológica}

\section{SUMMARY}

Objective: To evaluate the quality of the laboratory of cervical cytology for low and high grade SIL on the Cervical Pathology Unit of the Talcahuano`s Naval Hospital through the cytohistological correlation. Material and Method: Retrospective, descriptive and analytical study. Review of 422 clinical records of patients entered to the Cervical Pathology Unit of the Naval Hospital between 1994-2004. In 204 cases presented positive cytology for SIL, of them $83.3 \%$ had biopsy under colposcopic vision, and $75 \%$ biopsy by loop electrical excision procedure. As classification diagnoses were used the Bethesda system. Results: Significant difference between the average of age in the patients who have low and high grade SIL, as much for the group with biopsy under colposcopic vision as for the group with biopsy by loop electrical excision 
procedure, does not exist. In those with biopsy under colposcopic vision had cytohistological coincidence was $67.1 \%$ for low grade SIL, and $67.1 \%$ for high grade SIL. In those with biopsy by loop electrical excision procedure cytohistological coincidence was $59.7 \%$ for low grade SIL, and $73.7 \%$ for high grade SIL. It exists correlation between cytology for SIL and histopatologic study as much of biopsy made under colposcopic vision, as by loop electrical excision procedure $(p<0,001)$. Conclusion: The average of age of the patients who had a low grade SIL in both groups was superior to the national and international series. In the Cervical Pathology Unit of the Talcahuano's Naval Hospital the cytological screening is reliable for low and high grade SIL.

\section{KEY WORDS: Cytohistological correlation, SIL, cytological screening}

\section{INTRODUCCIÓN}

La citología cervico-vaginal es la técnica diagnóstica más efectiva para la prevención y detección de lesiones pre y cancerosas de cérvix (1). Sin embargo, actualmente todavía existe un cierto grado de discordancia en la correlación citohistológica. Por este motivo el Colegio Americano de Patólogos propone diversos métodos para el control de calidad en los laboratorios de citología (2, 3). Uno de estos métodos consiste en comparar los diagnósticos de las citologías y biopsias (4) con la finalidad de valorar la correlación citohistológica en los distintos grados de lesión intraepitelial escamosa así como determinar subdiagnósticos y sobrediagnósticos (5).

El objetivo de este estudio es evaluar la calidad del laboratorio de citología cervical del Hospital Naval de Talcahuano mediante el estudio de la correlación citohistológica para lesión intraepitelial de bajo y alto grado.

\section{MATERIAL Y METODO}

Estudio retrospectivo, descriptivo y analítico. Se revisaron 422 historias clínicas de pacientes ingresadas a la unidad de patología cervical del Hospital Naval de Talcahuano en el período comprendido entre los años 1994 y 2004. En 204 casos hubo citología positiva para lesión intraepitelial (LIE), y de estos, 170 (83,3\%) fueron sometidas al menos a una biopsia bajo visión colposcópica, y $153(75 \%)$ de las 204 pacientes tenían al menos

Tabla I

\section{EDAD DE LAS PACIENTES CON CITOLOGÍA POSITIVA PARA LIE Y CON BIOPSIA BAJO VISIÓN COLPOSCÓPICA}

\begin{tabular}{lccr}
\hline $\begin{array}{l}\text { Citología vs } \\
\text { colposcopia }\end{array}$ & Media (años) & Rango & $\pm D E$ \\
\hline LIE Bajo Grado & 34,5 & $17-64$ & 10,46 \\
LIE Alto Grado & 38,1 & $23-66$ & 9,39 \\
\hline
\end{tabular}

una biopsia por escisión con asa electroquirúrgica. Como clasificación diagnóstica se empleó el sistema Bethesda (6). Para el análisis estadístico se utilizó la prueba $\mathrm{t}$ de Student y la dócima no paramétrica Chi cuadrado de independencia.

\section{RESULTADOS}

La variable edad es analizada para ambos grupos en las Tablas I y II. No hubo diferencia significativa entre la media de edad en las pacientes que presentaban LIE de bajo y alto grado con citología positiva para LIE y con biopsia bajo visión colposcópica (Tabla I). No hubo diferencia significativa entre la media de edad en las pacientes que presentaban LIE de bajo y alto grado con citología positiva para LIE y con biopsia por escisión con asa electroquirúrgica (Tabla II).

Los resultados de los informes citológicos e informes histopatológicos de biopsias realizadas bajo visión colposcópica se detallan en la Tabla III. Hubo coincidencia citohistológica de $67,1 \%$ cuando el informe citológico dio como resultado una lesión intraepitelial de bajo grado $(n=88)$, y un $67,1 \%$ cuando el resultado fue lesión intraepitelial de alto grado $(n=82)$. De acuerdo a estos resultados, hubo correlación significativa entre el informe citológico para lesión intraepitelial y el informe histopatológico de biopsia realizada bajo visión colposcópica $(p<0,001)$.

Los resultados citológicos e histopatológicos se encuentran representados para el grupo de biopsia con asa electroquirúrgica en la Tabla IV.

Tabla II

\section{EDAD DE LAS PACIENTES CON CITOLOGÍA POSITIVA PARA LIE Y CON BIOPSIA POR ESCI- SIÓN CON ASA ELECTROQUIRÚRGICA}

\begin{tabular}{lccc}
\hline $\begin{array}{l}\text { Citología vs } \\
\text { escisión con Asa }\end{array}$ & Media (años) & Rango & $\pm D E$ \\
\hline LIE Bajo Grado & 34,2 & $17-62$ & 10,96 \\
LIE Alto Grado & 37,5 & $23-66$ & 10,49 \\
\hline
\end{tabular}


Tabla III

RESULTADOS DE CITOLOGÍA POSITIVA PARA LIE Y CON BIOPSIA BAJO VISIÓN COLPOSCÓPICA

\begin{tabular}{|c|c|c|c|c|c|c|}
\hline & Negativa & $\begin{array}{c}\text { LIE BG } \\
\text { HPV }\end{array}$ & $\begin{array}{c}\text { LIE BG } \\
\text { NIE I }\end{array}$ & $\begin{array}{c}\text { LIE AG } \\
\text { NIE II }\end{array}$ & $\begin{array}{c}\text { LIE AG } \\
\text { NIE III }\end{array}$ & Carcinoma \\
\hline \multicolumn{7}{|l|}{ LIE BG: } \\
\hline $\operatorname{HPV}(n=10)$ & 2 & 4 & 2 & 1 & 1 & 0 \\
\hline NIE I $(n=78)$ & 8 & 11 & 42 & 12 & 5 & 0 \\
\hline \multicolumn{7}{|l|}{ LIE AG: } \\
\hline NIE II $(n=39)$ & 6 & 1 & 6 & 14 & 12 & 0 \\
\hline NIE III $(n=43)$ & 6 & 0 & 5 & 7 & 22 & 3 \\
\hline Total & 22 & 16 & 55 & 34 & 30 & 3 \\
\hline
\end{tabular}

LIE BG: lesión intraepitelial de bajo grado.

LIE AG: lesión intraepitelial de alto grado.

NIE: neoplasia intraepitelial.

HPV: virus papiloma humano.

Hubo coincidencia citohistológica en 59,7\% cuando el informe citológico dio como resultado una lesión intraepitelial de bajo grado $(n=77)$, y de $73,7 \%$ cuando el resultado fue lesión intraepitelial de alto grado $(n=76)$. De acuerdo a estos resultados, hubo correlación significativa entre el informe citológico para lesión intraepitelial y el informe histopatológico de biopsia realizada con asa electroquirúrgica $(p<0,001)$.

\section{DISCUSIÓN}

En ambos grupos de estudio (citología con biopsia bajo visión coloposcópica y citología con biopsia por escisión por asa electroquirúrgica) el promedio de edad de los pacientes que portaban un LIE alto grado fue mayor que el grupo de pacientes que portaban un LIE bajo grado, pero sin diferencia estadísticamente significativa. El promedio de edad de los pacientes que portaban un LIE bajo grado en ambos grupos de estudio fue muy superior a la mayoría de las series reportadas tanto a nivel nacional e internacional (7-11).

La citología cervico-vaginal ha demostrado ser útil en el screening de las lesiones intraepiteliales tanto de bajo como alto grado, esto queda confirmado en este trabajo por el análisis estadístico aplicado al informe citológico de cada paciente,

Tabla IV

RESULTADOS DE CITOLOGÍA POSITIVA PARA LIE Y CON BIOPSIA POR ESCISIÓN CON ASA ELECTROQUIRÚRGICA

\begin{tabular}{|c|c|c|c|c|c|c|}
\hline & Negativa & $\begin{array}{c}\text { LIE BG } \\
\text { HPV }\end{array}$ & $\begin{array}{c}\text { LIE BG } \\
\text { NIE I }\end{array}$ & $\begin{array}{c}\text { LIE AG } \\
\text { NIE II }\end{array}$ & $\begin{array}{c}\text { LIE AG } \\
\text { NIE III }\end{array}$ & Carcinoma \\
\hline \multicolumn{7}{|l|}{ LIE BG: } \\
\hline $\operatorname{HPV}(n=4)$ & 1 & 0 & 2 & 1 & 0 & 0 \\
\hline NIE I $(n=73)$ & 9 & 2 & 42 & 13 & 7 & 0 \\
\hline \multicolumn{7}{|l|}{ LIE AG: } \\
\hline NIE ॥ $(n=33)$ & 2 & 0 & 5 & 11 & 14 & 1 \\
\hline NIE III $(n=43)$ & 2 & 0 & 6 & 6 & 25 & 4 \\
\hline Total & 14 & 2 & 55 & 31 & 46 & 5 \\
\hline
\end{tabular}

LIE BG: lesión intraepitelial de bajo grado.

LIE AG: lesión intraepitelial de alto grado.

NIE: neoplasia intraepitelial.

HPV: virus papiloma humano. 
frente al respectivo informe histopatológico, ya sea realizado mediante biopsia bajo visión colposcópica o biopsia realizada por escisión con asa electroquirúrgica.

Consideramos importante resaltar que en los casos en que se comparó informe citológico frente a biopsia obtenida bajo visión colposcópica se observó que en los LIE de alto grado hay un subdiagnóstico citológico en un $3,65 \%$ de los informes, lo mismo ocurre en un $6,57 \%$ en el grupo en que se comparo informe citológico frente a biopsia obtenida con asa electroquirúrgica. A pesar de la importancia clínica de estas disrrelaciones, la citología se mantiene como un buen método de screening de lesiones intraepiteliales de alto grado.

Es importante señalar que en ocasiones resulta difícil distinguir citológicamente una NIE II de una NIE III, para este último aspecto la utilización de la clasificación de Bethesda permitió obtener mayor correlación ya que estos casos fueron incluidos dentro de las lesiones intraepiteliales de alto grado lo cual concuerda con el trabajo realizados por Kurman y Solomon al respecto (6).

Los resultados obtenidos nos permiten afirmar que en la unidad de patología cervical del Hospital Naval de Talcahuano el screening citológico es confiable tanto para lesiones intraepiteliales de bajo y alto grado. De acuerdo a los resultados obtenidos en este trabajo como en otros trabajos previos $(12,13)$, la correlación citohistológica demostró que sigue siendo un buen método para el control de calidad interno en los servicios de anatomía patológica.

\section{BIBLIOGRAFÍA}

1. Anderson GH, Boyes DA, Benedet JL et al. Organisation and results of the cervical cytology screening programme in British Columbia, 1955-85. BMJ 1988; 296: 975-978.

2. Christopherson WM, Scott MA. Trends in mortality from uterine cancer in relation to mass screening. Acta Cytol 1977; 21: 5-9.

3. Carney CN. Quality control in cytopathology: a system for simultaneous monitoring of accuracy and education and for proficiency testing. Acta Cytol 1984; 28: 535-540

4. Van der Graaf Y, Vooijs GP. False negative rate in cervical cytology. J Clin Pathol 1987; 40: 438-42.

5. Commission on Laboratory Accreditation. 1994 Inspection Checklist: Cytopathology, Northfield. III: College of American Pathologist 1994; 08.4025, 08.4027, 08.4035, 08.4040.

6. Kurman RJ, Solomon D. The Bethesda System for reporting Cervical/Vaginal Citology Diagnoses. New York: Springer-Verlag; 1994.

7. Serman F. Cáncer cervicouterino: epidemiología, historia natural y rol del virus Papiloma Humano, perspectivas en prevención y tratamiento. Rev Chil Obstet Ginecol 2002; 67(4): 318-323.

8. Nazzal O, Reinero M, Abarzúa A, Liendo R, Palma C. Patología preinvasora del cervix. Rev Chil Obstet Ginecol 2003; 68(3): 189-196.

9. Richart RM. Natural history of cervical intraepithelial neoplasia. Clin Obstet Gynecol 1967; 10: 748-84.

10. Richart RM. Natural history of cervical intraepithelial neoplasia. Obstet Gynecol 1990; 75: 131-33.

11. Hellberg D, Nilsson S, Valentin J. Positive cervical smear with subsequent normal colposcopy and histology. Frequency of CIN in a long-term followup. Gynecol Oncol 1994; 53(2): 148-51.

12. US Department of Labor. Occupational Health and Safety Administration. Laboratory requirements. Condition: Cytology. Federal Register 1992; 57: 7169.

13. Tritz DM,Weekes JA, Spires SE, Sattich M, Banks $H$, Cibull LM, Davey DD. Etiologies for noncorrelating cervical cytologies and biopsies. Am J Clin Pathol 1995; 103(5): 594-597. 